\title{
A NOTE ON THE VERTEX-SWITCHING RECONSTRUCTION
}

\author{
I. KRASIKOV \\ School of Mathematical Sciences \\ Tel-Aviv University \\ Tel-Aviv \\ Israel
}

(Received October 7, 1987 and in revised form November 15, 1987)

ABSTRACT. Bounds on the maximum and minimum degree of a graph establishing its reconstructibility from the vertex switching are given. It is also shown that any disconnected graph with at least five vertices is reconstructible.

KEY WORDS AND PHRASES. Vertex-Switching, Reconstruction. 1980 AMS SUBJECT CLASSIFICATION CODE. $05 \mathrm{C06}$.

1. INTRODUCTION.

A switching $G_{v}$ of a graph $G$ at vertex $v$ is a graph obtained from $G$ by deleting all edges incident to $v$ and inserting all possible edges to $v$ which are not in $G$. Since switching is a commutative operation, i.e., $\left(G_{v}\right)_{u}=\left(G_{u}\right)_{v}$, the definition can be naturally extended to arbitrary subsets of the vertex set $V(G)$. Thus, $G_{A}$ is defined for all $A \subseteq V(G)$.

The Vertex-Switching Reconstruction Problem, proposed by Stanley [1], asks: Is $G$ uniquely determined up to isomorphism by the set (deck ), $\left\{G_{\nu}\right\}_{\nu} \in V(G)$ ? If the answer is "yes" then $G$ is called reconstructible.

It was shown in [1] that any graph $G$ with $n=|V(G)| \neq 0(\bmod 4)$ is reconstructible. It seems that a little is known about the case $n=0(\bmod 4)$. However, Stanley pointed out [1], that the degree sequence of a graph, and consequently, the number of edges easily reconstructible, provided $n \neq 4$. Bounds on the number of edges in a graph, e(G), establishing its reconstructibility was given [2]. Namely:

$$
\text { e(G) } \notin\left[\frac{n(n-2)}{4}, \frac{n^{2}}{4}\right], n \neq 4 \text {. }
$$

As might be expected, in virtue of the last result, $G$ is reconstructible if it has a vertex of degree not close to $n / 2$ or if $G$ is disconnected. Here we will prove the last claim (Theorem 2) and show that for sufficiently large $n$ a graph is reconstructible if $\max (\Delta, n-\delta)>0.9 n$, where $\Delta$ and $\delta$ are the maximum and the minimum degree of $G$ respectively. Actually, we prove a little more, namely: 
2. MAIN RESULTS.

THEOREM 1. If $\min \left(\mathrm{n}\left(\begin{array}{c}\mathrm{n}-1 \\ \Delta\end{array}\right), \mathrm{n}\left(\begin{array}{c}\mathrm{n}-1 \\ \delta\end{array}\right)\right)<2^{\mathrm{n} / 2-3}$,

then $G$ is reconstructible.

PROOF. In virtue of the quoted result of Stanley, we may assume $n=0(\bmod 4)$. We will consider a graph $G$ as a spanning subgraph of a fixed copy of the complete graph $K_{n}$. The switching equivalence class $G^{*}$ of $G$ is the set of all $\mathrm{H} \subset \mathrm{K}_{\mathrm{n}}$ isomorphic to $\mathrm{G}$ such that $H=G_{A}$ for some switching $A \subseteq V(G)$.

For each subgraph $g \subset G$, let $\mu\left(G^{*} \supset g\right)$ be the number of those elements of $G^{*}$ which contain a fixed copy of $\mathbf{g}$.

First we show that $G$ is reconstructible if

$$
\frac{\mu\left(G^{*} \supset g\right) s\left(g \rightarrow K_{n}\right)}{s(g \rightarrow G)}<2^{n / 2-2},
$$

where $s(H \rightarrow F)$ is the number of the subgraphs of $F$ isomorphic to $H$.

Observe that

$$
\left|G^{*}\right| s(g \rightarrow G) \leqq \mu\left(G^{*} \supset g\right) s\left(g \rightarrow K_{n}\right) \text {. }
$$

On the other hand, consider the set $S_{i}=\left\{A: G_{A} \in G^{*},|A|=i\right\}$. Observe that $\Sigma\left|S_{i}\right|=2\left|G^{*}\right|$ since $G_{A}$ and $G_{A}, \bar{A}=V(G) \backslash A$, are identical. It is known that for a nonreconstructible graph $\left|S_{4 i}\right| \geqq\left(\begin{array}{c}n / 2 \\ 2 i\end{array}\right)$ ([2], Corollary 2.4). Thus, if $\mathrm{G}$ is not reconstructible then

$$
2\left|G^{*}\right| \geqq \Sigma\left(\begin{array}{c}
n / 2 \\
2 i
\end{array}\right)=2^{n / 2-1}
$$

Comparing (2.2) and (2.3), we get that (2.1) is enough for the reconstructibility of G. Let now $g$ be a star $K_{1, \Delta^{*}}$ Observe that $\mu\left(G^{*} \supset K_{1, \Delta}\right) \leq 2$ since the only proper switching, possibly preserving a fixed copy of $\mathrm{K}_{1, \Delta}$, is $A=v\left(\mathrm{~K}_{1, \Delta}\right)$. Furthermore, $\mathbf{s}\left(\mathrm{g} \rightarrow \mathrm{K}_{\mathrm{n}}\right)=\mathrm{n}\left(\begin{array}{c}\mathrm{n}-1 \\ \Delta\end{array}\right)$. Hence, by $(2.1)$, $\mathrm{G}$ is reconstructible if $\mathrm{n}\left(\begin{array}{c}\mathrm{n}-1 \\ \Delta\end{array}\right)<2^{\mathrm{n} / 2-3}$. Now, to complete the proof, one has to consider the complementary graph $\bar{G}$, which is reconstructible iff $\mathrm{G}$ is. $\square$

Now we will prove that disconnected graphs are reconstructible. First we need the following simple lemma:

LEMMA 1. Suppose that nonisomorphic graphs $G$ and $H$ have the same deck. Then for any $v \in V(G)$ there is $u \in V(G), \nu \neq u$, such that $G_{v u} \cong H$.

PROOF. Since the decks of $G$ and $H$ are equal then there is a bijection $\phi: V(G) \rightarrow V(H)$ such that $G_{v} \equiv H_{\phi(\nu)}$. Let $h_{\nu}: H_{\phi(\nu)} \rightarrow G_{\nu}$ be an isomorphism. Choosing $u=h(\phi(\nu))$ we obtain $G_{\nu u} \cong H$. Moreover, since $G_{\nu \nu}=G$, then $\nu \neq \phi(\nu)$. 口 
COROLLARY 1. Let $n \neq 4$. If $G_{v u}$ and $G, \nu \neq u$, have the same deck then $\operatorname{deg}(v)+\operatorname{deg}(u)=n$ or $n-2$, depending on whether $\nu$ and $u$ are adjacent in $G$ or are not. PROOF. Let $e(v, u)$ be the number of edges between $v$ and $u$. Since $e(G)=e(H)$ then

$$
\operatorname{deg}(v)+\operatorname{deg}(u)-2 e(v, u)=\frac{1}{2} \cdot 2(n-2)=n-2 \cdot 0
$$

COROLLARY 2. If $\mathrm{G}$ is not reconstructible and $\mathrm{n} \neq 4$ then $n-2 \leq \delta+\Delta \leq n$.

PROOF. This easily follows from Lemma 1 and Corollary 1 . We omit the details.

THEOREM 2. Any disconnected graph is reconstructible, provided $n \neq 4$.

PROOF. Assume the contrary. Then there are two nonisomorphic graphs $G$ and $H$ with the same deck, $n \neq 4$, and, say, $G$ is disconnected. Denote by $C$ a minimal connected component of $G$. First we show that $G$ has exactly two connected components and $C \cong K_{\delta+1}$.

Let $v$ be a vertex of the minimal degree in $C$, and let $u$ be such a vertex that $G_{v u} \cong H$. We claim that either $u=\phi(\nu) \in \bar{C}$ or $G$ is regular of degree $\frac{n-2}{2}$. Indeed, otherwise,

$$
|C| \geq \max (\operatorname{deg}(v)+1, \operatorname{deg}(u)+1)>n / 2 \text {, }
$$

which contradicts the minimality of $\mathrm{C}$. Furthermore, if $\mathrm{G}$ is regular then again $\nu$ and $u$ are in different components since, otherwise, the degree sequences of $G$ and $G_{v u}$ are different. Now it follows by Corollary $1, \operatorname{deg}(v)+\operatorname{deg}(u)=n-2$. Therefore, $G$ has exactly two components, $C$ is regular, and $\Delta \geq n / 2$.

Let us show that $C$ is just $k_{\delta+1}$. Since all vertices of degree $\Delta$ are in $\bar{C}$, we have

$$
\operatorname{deg}(v)+1 \leqq|C| \leqq n-\Delta-1 \text {. }
$$

Hence, applying Corollary 2, we get

$$
\mathrm{n}-2 \leqq \delta+\Delta \leqq \operatorname{deg}(\nu)+\Delta \leqq \mathrm{n}-2 \text {. }
$$

Thus, $\operatorname{deg}(v)=\delta, \operatorname{deg}(u)=\Delta$, and $C \cong K_{\delta+1}$.

Finally, $G_{v u} \cong G$ since $\operatorname{deg}(v)=|C|-1, u \in \bar{C}$ and $\operatorname{deg}(u)=\Delta=|\bar{C}|-1$, which.is a contradiction. This completes the proof. 口

\section{REFERENCES}

1. STANLEY, R.P. Reconstruction from vertex switching. J. Combin. Theory (B) 38, (1985), 132-138.

2. KRASIKOV, I. and RODITTY, Y. Balance equations for reconstruction problems. Archiv der Mathematik, Vol. 48 (1987), 458-464. 


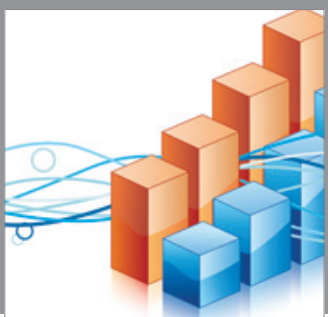

Advances in

Operations Research

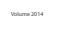

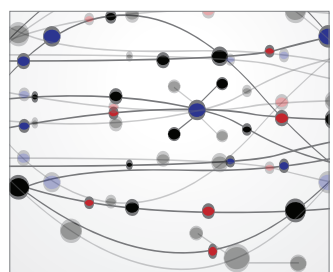

\section{The Scientific} World Journal
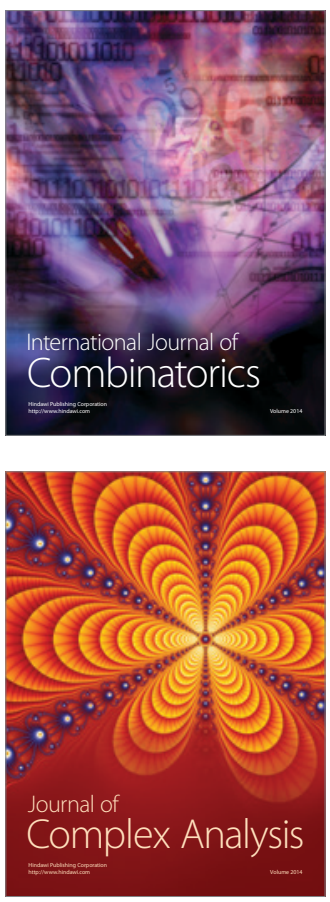

International Journal of

Mathematics and

Mathematical

Sciences
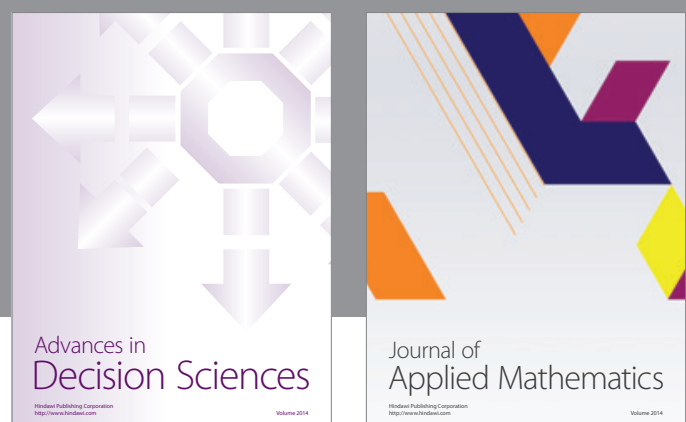

Journal of

Applied Mathematics
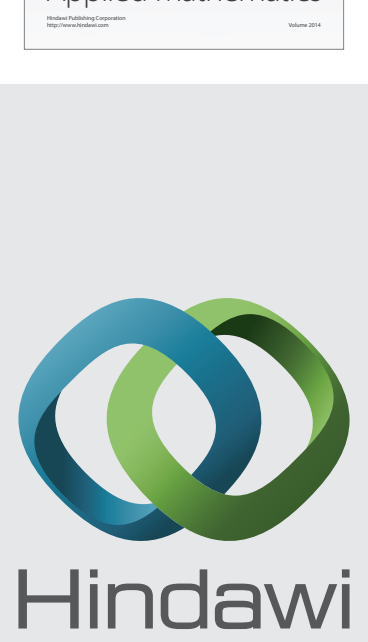

Submit your manuscripts at http://www.hindawi.com
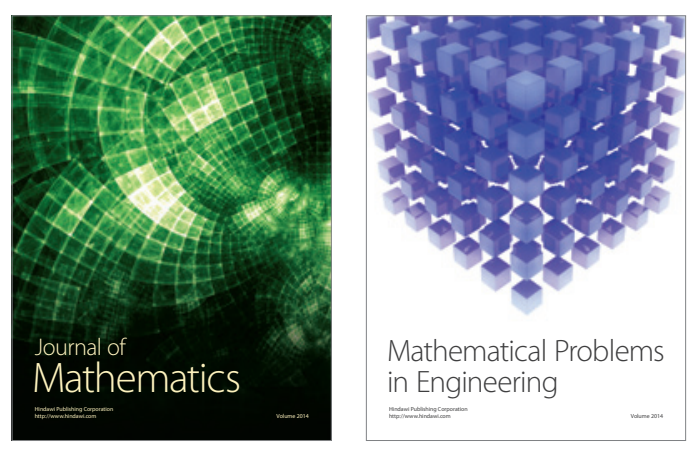

Mathematical Problems in Engineering
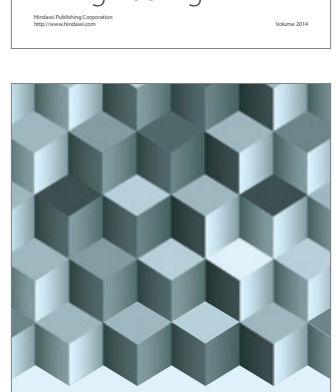

Journal of

Function Spaces
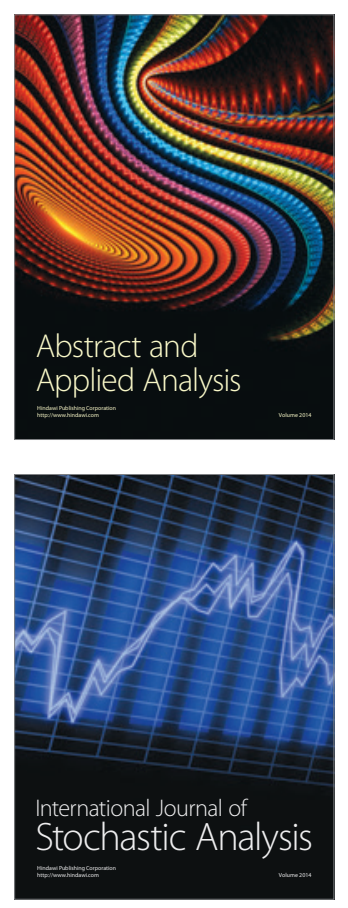

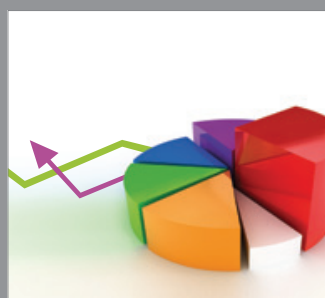

ournal of

Probability and Statistics

Promensencen
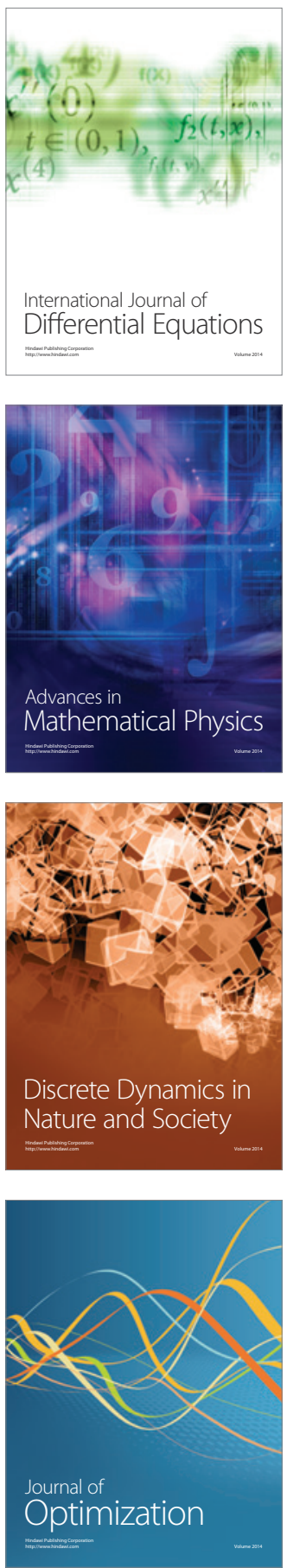